\title{
Concordância interobservador na avaliação de lesões do tendão subescapular usando imagens de Ressonância Nuclear Magnética
}

\author{
Interobserver agreement in the assessment of subscapularis tendon injuries using \\ Magnetic Nuclear Resonance imaging \\ Concordancia interobservadora en la evaluación de lesiones del tendón subescapular usando \\ imágenes de Resonancia Nuclear Magnética
}

\author{
Aleixo Abreu TANURE \\ Roberto Barreto MAIA ${ }^{2}$ \\ Thiago Alencar FORTALEZA ${ }^{3}$ \\ Luis Guilherme Rosifini ALVES REZENDE ${ }^{4}$ \\ Adriana Vieira Pedreira CABRAL ${ }^{5}$ \\ Rogério Meira BARROS ${ }^{6}$
}

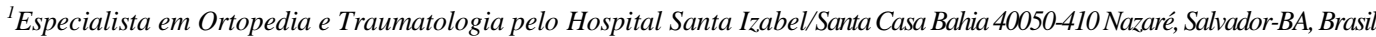
${ }^{2}$ Ortopedista, Membro do Grupo do Ombro e Cotovelo do Hospital Santa Izabel/Santa Casa Bahia 40050-410 Nazaré, Salvador-BA, Brasil

${ }^{3}$ Especialista em Cirurgia do Ombro e Cotovelo pelo Hospital Santa Izabel/Santa Casa Bahia 40050-410 Nazaré, Salvador-BA, Brasil

${ }^{4}$ Especialista em Ortopedia e Traumatologia pelo Hospital das Clínicas da Faculdade de Medicina de Ribeirão Preto da Universidade de São Paulo (USP); Residente, Cirurgia da Mão, Hospital das Clínicas da Faculdade de Medicina de Ribeirão Preto da Universidade de São Paulo (USP);

Mestrando Programa de Pós-Graduação Ciências da Saúde Aplicadas ao Aparelho Locomotor/ Faculdade de Medicina de Ribeirão Preto da Universidade de São Paulo (USP) 14049-900 Ribeirão Preto-SP, Brasil

${ }^{5}$ Radiologista do Image Memorial Laboratório e Imagem 40170-110 Ondina, Salvador-BA, Brasil

${ }^{6}$ Ortopedista, Mestre em Ciências, Área de Ortopedia e Traumatologia, Faculdade de Medicina de São Paulo da Universidade de São Paulo (USP; Presidente da SBOT-BA (Sociedade Brasileira de Ortopedia e Traumatologia) Regional Bahia;

Chefe do Grupo do Ombro e Cotovelo, Preceptor da Residência Médica de Ortopedia e Trauma, Hospital Santa Izabel-Santa Casa40050-410 Salvador-BA, Brasil

\section{Resumo}

Introdução: As lesões do tendão musculo subescapular (TMSE) do manguito rotador passaram a ter aumento de sua incidência com as avançadas técnicas de diagnostico por imagem e artroscópicas. A Ressonância Nuclear Magnética (RM) apresenta boa sensibilidade e especificidade permitindo adequada acurácia diagnóstico. Objetivo: avaliação da concordância interobservador da RM para Lesões do TMSE comparada à artroscopia. Métodologia: 49 pacientes sintomáticos foram submetidos à RM para diagnóstico de lesão do TMSE e avaliação da Classificação de Lafosse, tendo sua concordância avaliada através da Artroscopia diagnostica. Resultados: A acurácia global foi $68 \%$ para concordância diagnóstica da Lesão do TMSE e 57,1\% para concordância na classificação de Lafosse. Discussão: obteve-se concordância para o diagnóstico de Lesão ou ausência de Lesão do TMSE de 0,49 e a concordância para a Classificação de Lafosse foi de 0,30. Conclusão: o diagnóstico da lesão do TMSE através da RM é de difícil realização.

Descritores: Manguito Rotador; Artroscopia; Imagem por Ressonância Magnética.

\section{Abstract}

Introduction: Injuries of subscapular muscle tendon (SEMT) of the rotator cuff have been increased in incidence with advanced imaging and arthroscopic techniques. Magnetic Nuclear Resonance (MRI) presents good sensitivity and specificity allowing adequate diagnostic accuracy. Objective: to evaluate the interobserver agreement of MRI for SEMT lesions compared to arthroscopy. Methods: 49 symptomatic patients underwent MRI for diagnosis of SEMT lesion and evaluation of the Lafosse Classification, and their concordance was assessed through diagnostic arthroscopy. Results: The overall accuracy was 68\% for diagnostic concordance of the SEMT Lesion and 57.1\% for concordance in the Lafosse classification. Discussion: concordance was obtained for the diagnosis of Lesion or absence of Lesion of the SEMT of 0.49 and the agreement for the Lafosse Classification was 0.30. Conclusion: the diagnosis of SEMT lesion through MRI is difficult to perform.

Descriptors: Rotator Cuff; Arthroscopy; Magnetic Resonance Imaging.

\section{Resumen}

Introducción: Las lesiones del tendón musculo subescapular (TMSE) del manguito de los rotadores pasaron a tener aumento de su incidencia con las avanzadas técnicas de diagnóstico por imagen y artroscópicas. La Resonancia Magnética Nuclear (RM) presenta buena sensibilidad y especificidad permitiendo una adecuada exactitud diagnóstica. Objetivo: evaluación de la concordancia entre observadores de la imagen RM para Lesiones del TMSE comparada a la artroscopia. Métodos: 49 pacientes sintomáticos fueron sometidos a la RM para diagnóstico de lesión del TMSE y evaluación de la Clasificación de Lafosse, teniendo su concordancia evaluada a través de la Artroscopia diagnóstica. Resultados: La exactitud global fue $68 \%$ para concordancia diagnóstica de la Lesión del TMSE y el $57,1 \%$ para concordancia en la clasificación de Lafosse. Discusión: se obtuvo concordancia para el diagnóstico de Lesión o ausencia de Lesión del TMSE de 0,49 y la concordancia para la Clasificación de Lafosse fue de 0,30. Conclusión: el diagnóstico de la lesión del TMSE a través de la RM es de difícil realización.

Descriptores: Manguito de los Rotadores; Artroscopía; Imagen por Resonancia Meganética.

\section{INTRODUÇÃO}

A primeira descrição da rotura do tendão subescapular é tradicionalmente atribuída ao estudo post-mortem de Smith et al. ${ }^{1}$, e esta lesão tem ganhado certa importância na literatura ortopédica. A rotura do tendão subescapular é rara em pacientes com lesão do manguito rotador, com uma prevalência estimada em 2 a $8 \% \%^{2-4}$. Apesar de ser descrita como uma ruptura infrequente, estudos recentes em pacientes submetidos a cirurgia artroscópica vêm demostrando prevalência bem maior deste tipo de lesão, estimando sua presença em 41 a $43 \%$ dos casos de rupturas do manguito rotador ${ }^{5-7}$. Esta discrepância pode em parte ser explicada pelo surgimento das novas técnicas cirúrgicas que permitem melhor visualização da lesão, entretanto deve-se considerar também a possibilidade de falhas diagnósticas durante a avaliação do manguito rotador.

O diagnóstico das lesões do subescapular permanece um desafio clínico considerável a despeito do crescente interesse dos ortopedistas por esta 
patologia. Os testes clínicos mais utilizados na investigação de lesões do subescapular são: lift-off test, internal rotation lag sign, e o belly-press test. Entre estes, o belly-press test é considerado como o de maior acurácia, tendo baixa sensibilidade $(27.8 \%)$, porém excelente especificidade $(100 \%)^{8}$. Devido à baixa sensibilidade dos testes clínicos, métodos de auxilio diagnóstico como radiografia simples, ultrassonografia, tomografia computadorizada com injeção de contraste intra-articular e ressonância magnética (RM) com ou sem contraste também são utilizados rotineiramente para complementar a avaliação diagnóstica.

A ressonância magnética é um dos exames mais estudados e aceitos no diagnóstico das patologias do ombro, especialmente nas doenças do manguito rotador ${ }^{6,9,10}$. Sua sensibilidade varia de 84 a $100 \%$ e especificidade de 77 a $97 \%{ }^{11}$. Apesar de sua excelente acurácia global, estudos que avaliaram o uso da RM para lesões do tendão subescapular têm mostrado resultados variados; nos quais a sensibilidade variou de $36 \%$ a $80 \%$ enquanto a especificidade alcançou 91 a $100 \% \%^{5,6,12}$.

A despeito do interesse crescente pela acurácia da ressonância magnética em diagnosticar roturas do músculo subescapular, atribui-se que erros de interpretação sejam comuns devido à baixa prevalência de lesão associada e à pequena experiência dos examinadores. A acurácia da RM é um produto direto da capacidade do observador, entretanto poucos estudos avaliaram a associação da concordância dos examinadores no diagnóstico das lesões do subescapular por ressonância magnética. Esta análise reveste-se de importância fundamental para melhorar o diagnóstico e o processo de tomada de decisão para uma abordagem terapêutica cirúrgica ou conservadora nos pacientes que apresentam lesões do subescapular, associadas a patologias do manguito rotador $^{13-15}$.

O objetivo do presente estudo é avaliar o efeito da concordância interobservador da ressonância magnética na acurácia diagnóstica das lesões do tendão do músculo subescapular isoladas ou em conjunto com lesões do manguito rotador, comparando-se com a visão direta dos achados da cirurgia artroscópica.

\section{MATERIAL E MÉTODO}

Foi realizado estudo de confiabilidade diagnóstica para avaliação da concordância interobservador da RM em lesões do tendão do músculo subescapular. Foram incluídos no estudo pacientes atendidos no Serviço de Cirurgia do Membro Superior do Hospital Santa Izabel, Salvador, Bahia, no período de julho de 2014 a novembro de 2014. O critério de elegibilidade para inclusão no estudo foi a presença de lesão do manguito rotador com indicação de cirurgia artroscópica. Não foram incluídos paciente que já haviam realizado cirurgia no ombro previamente ao estudo, devido a maior dificuldade técnica para detectar as lesões ao exame da ressonância magnética. $O$ estudo seguiu os preceitos de ética estabelecidos para pesquisas envolvendo seres humanos (Resolução no 466/2012 do CNS) e foi aprovado pelo Comitê de Ética em Pesquisa do Hospital Santa Izabel em Salvador, Bahia. Todos os pacientes envolvidos na pesquisa assinaram o Termo de Consentimento Livre e Esclarecido após serem informados de todas as etapas envolvidas na pesquisa.

Todos os indivíduos selecionados para o trabalho tiveram suas imagens de RM do ombro em estudo avaliadas por três examinadores: um especialista em cirurgia do ombro experiente com mais de 50 artroscopias realizadas ao ano; um radiologista especializado em imaginologia musculoesquelética; e um ortopedista em processo de especialização na cirurgia de ombro (fellow). Para simular a realidade clínica cotidiana, os exames analisados foram realizados em instituições especializadas em bioimagem de distintas origens dentro do estado da Bahia. As imagens foram analisadas previamente por dois especialistas em cirurgia de ombro e um radiologista (diferentes dos examinadores) para garantir que a qualidade dos filmes fosse adequada e foram excluídos os exames considerados tecnicamente insuficientes.

As imagens foram selecionadas e entregues sequencialmente a cada examinador. Os médicos analisaram as imagens separadamente em momentos distintos e não tinham acesso aos dados clínicos dos pacientes, exceto o conhecimento que eles tinham alguma patologia no manguito rotador. Todos os examinadores preencheram um questionário específico da pesquisa, no qual era registrado a presença ou não de lesão do tendão subescapular e a classificação da lesão como descrita por Lafosse ${ }^{16}$.

Utilizou-se estatística descritiva para apresentação global dos dados. A estatística kappa foi utilizada para medir a concordância interobservador entre os três médicos. Esta é uma medida de concordância usada em escalas nominais que fornece uma ideia do grau de concordância de dois ou mais observadores na interpretação de um determinado resultado. Para análise foram observados os critérios de Landis e $\mathrm{Koch}^{17}$ que consideram concordância quase perfeita quando o índice está entre 0,81 e 1,00, substancial entre 0,61 e 0,80 , moderada entre 0,41 e 0,60 , razoável entre 0,21 e 0,40 , e pobre quando menor que 0,20 . Em adição, foram calculadas as respectivas acurácias dos examinadores quando comparadas à artroscopia.

\section{RESULTADOS}

Foram coletados dados de 49 pacientes ao longo do período de julho a novembro de 2014. Os 
pacientes tinham em média 57,9 anos $( \pm 7,7)$, sendo o paciente mais novo com 38 e o mais velho com 76 anos. Havia no estudo $25(51 \%)$ pacientes do sexo masculino e 24 (49\%) pacientes do sexo feminino. O ombro direito foi acometido em 30 casos $(61,2 \%)$ enquanto o esquerdo representou $19(38,8 \%)$.

Analisando a confiabilidade do método, os resultados apontam uma concordância com índice de kappa de 0,49 em diferenciar a presença ou não de lesão no tendão do músculo subescapular, o que corresponde a uma concordância moderada. Ao analisar a concordância em relação ao grau de lesão segundo a classificação de Lafosse et al. ${ }^{16}$, o índice kappa baixou para 0,30 o que corresponde a uma concordância apenas razoável.

Quando validada a interpretação dos exames com o diagnóstico artroscópico, as classificações conferidas pelos examinadores baseada apenas na presença ou ausência de lesão, nota-se que o especialista tende a superestimar, enquanto que o fellow e o radiologista tendem a subestimar o número de lesões. Na classificação por tipo, o especialista superestima as lesões do tipo 1, o radiologista subestima todas as lesões e o fellow subestima apenas as lesões do tipo 2 (Tabela 1).

Tabela 1. Classificação da lesão pelos examinadores em comparação com os achados artroscópicos

\begin{tabular}{|c|c|c|}
\hline Examinador & $\begin{array}{c}\text { Presença } \\
\text { da lesão (\%) }\end{array}$ & $\begin{array}{c}\text { Tipo } \\
\text { de lesão (\%) }\end{array}$ \\
\hline Especialista & $32(65,3 \%)$ & $\begin{array}{l}\text { Tipo } 1-27(55,1 \%) \\
\text { Tipo } 2-4(8,2 \%) \\
\text { Tipo } 3-1(2 \%)\end{array}$ \\
\hline Radiologista & $17(34,7 \%)$ & $\begin{array}{l}\text { Tipo } 1-10(20,4 \%) \\
\text { Tipo } 2-3(6,1 \%) \\
\text { Tipo } 3-4(8,2 \%)\end{array}$ \\
\hline Fellow & $23(46,9 \%)$ & $\begin{array}{l}\text { Tipo } 1-18(36,7 \%) \\
\text { Tipo } 2-1(2 \%) \\
\text { Tipo } 3-4(8,2 \%)\end{array}$ \\
\hline Artroscopia & $27(55,1 \%)$ & $\begin{array}{l}\text { Tipo } 1-18(36,7 \%) \\
\text { Tipo } 2-5(10,2 \%) \\
\text { Tipo } 3-4(8,2 \%)\end{array}$ \\
\hline
\end{tabular}

Com base nesses dados, foi encontrada uma acurácia de $69,4 \%$ para o especialista, $59,2 \%$ para o radiologista e $75,5 \%$ para o fellow, resultando numa acurácia global de 68\%. (Tabela 2).

Quando a avaliação é feita pela classificação de Lafosse et al. ${ }^{16}$ notamos que o fellow e o especialistas concluem o diagnóstico de maneira estatisticamente significante, enquanto o radiologista não alcança significância estatística. $\mathrm{O}$ especialista acertou 54,5\% dos tendões íntegros e $72 \%$ dos tendões com lesão tipo 1; O radiologista acertou $77,3 \%$ dos tendões íntegros e $11,1 \%$ dos tendões com lesão tipo 1 ; O fellow acertou $81,8 \%$ dos tendões íntegros e 61,1\% dos tendões com lesão tipo 1. Novamente o fellow foi o melhor examinador entre os três. Neste caso, as acurácias dos examinadores encontram-se entre 44,9\% e 67,3\%, com acurácia global de $57,1 \%$. (Tabela 3 ).

Tabela 2. Comparação da concordância dos examinadores com a presença de lesão artroscópica

\begin{tabular}{c|l|l|l|l|l}
\hline Examinador & Íntegro & Lesado & Kappa & Acurácia* $^{*}$ & $\mathbf{p}$ \\
\hline $\begin{array}{c}\text { Especialista } \\
\text { Integro } \\
\text { Lesado }\end{array}$ & 12 & 5 & 0,368 & $69,4 \%$ & 0,008 \\
$\begin{array}{c}\text { Radiologista } \\
\text { Întegro } \\
\text { Lesado }\end{array}$ & 17 & 15 & 0,208 & $59,2 \%$ & 0,112 \\
$\begin{array}{l}\text { Fellow } \\
\text { Íntegro }\end{array}$ & 18 & 8 & 0,513 & $75,5 \%$ & $<0,001$ \\
$\quad$ Lesado & 19 & & & \\
\hline *Acurácia global de 68,0\% & & & & & \\
\hline
\end{tabular}

Tabela 3. Concordância dos examinadores quando considerada o tipo da lesão segundo Lafosse et al em comparação com os achados artroscópicos

\begin{tabular}{llllc}
\hline Examinador & Acertos & Kappa & Acurácia* & P \\
Especialista & 29 & 0,354 & $59,2 \%$ & $<0,001$ \\
Radiologista & 22 & 0,110 & $44,9 \%$ & 0,232 \\
Fellow & 33 & 0,472 & $67,3 \%$ & $<0,001$ \\
\hline
\end{tabular}

*Acurácia global de $57,1 \%$

\section{DISCUSSÃO}

A utilidade de um teste diagnóstico depende da sua acurácia e da sua confiabilidade. A acurácia é reflexo da sensibilidade e especificidade inerente ao teste, enquanto a confiabilidade é a capacidade do teste produzir os mesmos resultados com a aplicação repetida ou quando interpretado por examinadores diferentes. Avaliação da concordância interobservador é uma parte importante na determinação da confiabilidade de um teste de diagnóstico $^{14}$. Quanto maior o contraste entre um resultado positivo e um resultado negativo melhor é a concordância interobservador. Isto irá afetar a acurácia do teste, estabelecendo um limite superior para a sua validade. Entretanto, quanto maior o espectro de variações que um teste possua, menor será a concordância entre os observadores. A ressonância magnética é um exame com grande detalhamento de imagens que permite uma excelente avaliação de patologias do ombro, mas que por outro lado permite um amplo espectro de interpretação da imagem fornecida, o que se reflete em uma variabilidade elevada para os observadores.

Nosso estudo teve como objetivo avaliar a concordância interobservador da análise de imagens de ressonância magnética entre um cirurgião de ombro experiente, um ortopedista em processo de especialização em cirurgia do ombro e um radiologista musculoesquelético. A concordância em diferenciar a presença ou não de lesão no tendão do músculo subescapular encontrada foi de 0,49 e a 
concordância de acordo com o grau de lesão segundo a classificação de Lafosse et al. ${ }^{16}$ ficou em 0,30. Essa diferença pode, portanto, ser reflexo do maior espectro no qual a lesão pode ser classificada. A acurácia variou de $59,2 \%$ a $75,5 \%$ em diferenciar a presença ou não de lesão; e variou de 44,9\% e 67,3\% em classificar a lesão segundo Lafosse. Estes valores estão abaixo dos níveis encontrados para o diagnóstico das lesões do supraespinhal, possivelmente por terem sofrido o efeito da confiabilidade também inferior.

Embora muitos estudos analisando a acurácia da ressonância magnética sem contraste em detectar lesões do manguito rotador tenham sido realizados, poucos deles analisaram a concordância interobservador na avaliação das lesões do manguito rotador, e nenhum especificamente das lesões do subescapular ${ }^{14,18-20}$.

O estudo de Spencer et al. ${ }^{14}$ mostrou uma concordância com índice kappa de 0,77 na diferenciação entre rotura completa e parcial de tendões do manguito rotador, de 0,55 em quantificar o número de tendões envolvidos e de 0,44 na avaliação do grau de retração ${ }^{13}$. Roberston et al. ${ }^{19}$ encontraram concordância substancial entre quatro radiologistas avaliando 97 pacientes para lesões completas e concordância pobre para lesões parciais $^{19}$. Balich et al. ${ }^{18}$ em um estudo com 5 radiologistas e 222 pacientes, corroboraram com esses achados, ao encontrar novamente concordância substancial para lesões completas e pobre para lesões parciais. Singson et al. ${ }^{20}$ encontraram melhor concordância ao comparar dois radiologistas musculoesqueléticos em 177 pacientes, com concordância quase perfeita para lesões completas e boa para lesões parciais. Contudo, cumpre destacar que esses estudos não avaliaram especificamente o tendão subescapular.

Em um estudo com desenho similar ao nosso, Szymanski et al. ${ }^{21}$ analisaram, dentre outros aspectos, a concordância interobervador entre um cirurgião de ombro experiente, um residente de ortopedia e um radiologista musculoesquelético sobre lesões especificas para o músculo subescapular. Entretanto, o estudo foi realizado analisando artrografia tomográfica, um exame pouco utilizado no nosso meio, por ser invasivo e com exposição a radiação. A concordância foi calculada através do coeficiente de concordância de Fermanian, e se mostrou boa (entre $0,5$ e 0,6$)$ ao considerar como parâmetros se o tendão se encontrava intacto, com delaminação ou rotura ${ }^{21}$. A validade dos examinadores quando comparados à artroscopia encontrada ficou entre 0,38 a 0,47 , sendo os piores resultados em diferenciar tendões intactos de delaminações tendinosas.

Ao comparar nosso estudo com os estudos de Balich et al. ${ }^{18}$, Robertson et al. ${ }^{19}$ e Singson et al. ${ }^{20}$ a concordância encontrada foi menor. As hipóteses incluem $\mathrm{o}$ fato do nosso estudo avaliar apenas o subescapular, que tem uma prevalência de lesão menor quando comparado ao musculo supraespinhal, associando a menor familiaridade dos cirurgiões e radiologistas em analisá-lo. Estes achados também são compatíveis com diversos estudos que apontam uma menor acurácia na RM em detectar lesões no músculo subescapular quando comparado com lesões do supraespinhal ${ }^{5,6,9,10,12}$.

Em relação ao estudo de Szymanski et al. ${ }^{21}$, a menor concordância observada pelo nosso estudo pode refletir o uso de métodos diagnósticos distintos. Apesar da injeção de contraste articular ser mais invasiva, este exame é melhor para avaliar lesões parciais do tendão. Outro ponto a ser considerado é que a concordância no estudo de Szymanski et al. ${ }^{21}$ apenas diferenciou entre tendão intacto, tendão com delaminação e rotura do tendão, enquanto que o presente estudo diferenciou entre tendão intacto e os cinco graus de lesão da classificação de Lafosse. Classificações com menos variáveis tendem a ter maior concordância interobservador. A validade encontrada em nosso estudo foi, no global, similar ao estudo de Szymanski et al. $^{21}$, exceto para o radiologista que apresentou concordância de 0,21 entre a ressonância magnética e a artroscopia.

$\mathrm{O}$ ortopedista fellow apresentou maior concordância com a artroscopia enquanto o radiologista apresentou uma tendência em subestimar o número de lesões e o especialista teve tendência de superestimá-las. Vale a pena ressaltar que apesar de superestimar o número de lesões, especialmente para lesões grau $\mathrm{I}$, isto não acarretaria realização de cirurgias desnecessárias pelo especialista pois a amostragem deste estudo consiste de pacientes que já haviam indicações ao procedimento artroscópico por outra patologia no manguito rotador, em sua maioria lesões do supraespinhal. Entretanto, este fato adverte cirurgiões para terem cautela ao indicarem cirurgias para lesões grau I isoladas do subescapular.

\section{CONCLUSÃO}

Nossos achados permitiram concluir que a confiabilidade diagnóstica da ressonância magnética para lesões do músculo subescapular é moderada para diferenciar a presença ou ausência de lesão $(k=0,49)$ e apenas razoável para a classificar a lesão segundo Lafosse $(k=0,30)$, com acurácia global de $68 \%$ e $57 \%$ quando validada pela artroscopia, respectivamente. O diagnóstico por imagem por meio da ressonância magnética para as lesões do subescapular apresenta variabilidade em suas interpretações, relacionados a qualidade do exame e experiência do avaliador.

\section{REFERÊNCIAS}

1. Ticker JB, Warner JJ. Single-tendon tears of the rotator cuff: evaluation and treatment of subscapularis tears and principles of treatment for 
supraspinatus tears. Orthop Clin North Am. 1997; 28(1):99-116.

2. Codman EA. Rupture of the supraspinatus tendon and other lesions in or about the subacromial bursa. The Shoulder. 2nd Ed. Boston: Thomas Todd; 1934. p. 262-312.

3. Deutsch A, Altchek DW, Veltri DM, Potter HG, Warren RF. Traumatic tears of the subscapularis tendon. Clinical diagnosis, magnetic resonance imaging findings, and operative treatment. Am J Sports Med. 1997;25(1):13-22.

4. Li XX, Schweitzer ME, Bifano JA, Lerman J, Manton GL, El-Noueam KI. MR evaluation of subscapularis tears. J Comput Assist Tomogr. 1999;23(5):713-17.

5. Adams CR, Schoolfield JD, Burkhart SS. Accuracy of preoperative magnetic resonance imaging in predicting a subscapularis tendon tear based on arthroscopy. Arthroscopy. 2010;26(11):1427-33.

6. Adams CR, Brady PC, Koo SS, Narbona P, Arrigoni P, Karnes GJ et al. A systematic approach for diagnosing subscapularis tendon tears with preoperative magnetic resonance imaging scans. Arthroscopy. 2012; 28(11):1592-600.

7. Pfirrmann CWA, Zanetti M, Weishaupt D, Gerber C, Hodler J. Subscapularis tendon tears: Detection and grading at MR ar- thrography. Radiology. 1999; 213:709-714.

8. Yoon JP, Chung SW, Kim SH, Oh JH. Diagnostic value of four clinical tests for the evaluation of subscapularis integrity. J Shoulder Elbow Surg. 2013;22(9):1186-92.

9. Beltran J. The use of magnetic resonance imaging about the shoulder. J Shoulder Elbow Surg. 1992; 1(6):321-33.

10.Houtz CG, Schwartzberg RS, Barry JA, Reuss BL, Papa L. Shoulder MRI accuracy in the community setting. J Shoulder Elbow Surg. 2011; 20(4):537-42.

11.Burks RT, Crim J, Brown N, Fink B, Greis PE. A prospective randomized clinical trial comparing arthroscopic single- and double-row rotator cuff repair: magnetic resonance imaging and early clinical evaluation. Am J Sports Med. 2009;37(4):674-82.

12. Gyftopoulos S, O' Donnell J, Shah NP, Goss J, Babb J, Recht MP. Correlation of MRI with arthroscopy for the evaluation of the subscapularis tendon: a musculoskeletal division's experience. Skeletal Radiol. 2013;42(9):1269-75.

13.Pfirrmann CW1, Zanetti M, Weishaupt D, Gerber C, Hodler J. Subscapularis tendon tears: detection and grading at MR arthrography. Radiology. 1999;213(3):709-14.
14.Spencer EE Jr, Dunn WR, Wright RW, Wolf BR, Spindler KP, McCarty $\mathrm{E}$ et al. Interobserver agreement in the classification of rotator cuff tears using magnetic resonance imaging. Am J Sports Med. 2008;36(1):99-103.

15.Toussaint B, Barth J, Charousset C, Godeneche A, Joudet $\mathrm{T}$, Lefebvre $\mathrm{Y}$ et al. New endoscopic classification for subscapularis lesions. Orthop Traumatol Surg Res. 2012;98(8 Suppl):S186-92.

16.Lafosse L, Jost B, Reiland Y, Audebert S, Tousaint B, Gobezie R. Structural integrity and clinical outcomes after arthroscopic repair of isolated subescapularis tears. J Bone Joint Surg Am. 2007;89(6):1184-93.

17.Landis JR, Koch GG. The measurement of observer agreement for categorical data. Biometrics. 1977;33(1):159-74.

18. Balich SM, Sheley RC, Brown TR, Sauser DD, Quinn SF. MR imaging of the rotator cuff tendon: interobserver agreement and analysis of interpretive errors. Radiology. 1997; 204(1):191-94.

19. Robertson PL, Schweitzer ME, Mitchell DG, Schlesinger F, Epstein RE, Frieman BG et al. Rotator cuff disor- ders: interobserver and intraobserver variation in diagnosis with MR imaging. Radiology. 1995;194(3):831-35.

20.Singson RD, Hoang T, Dan S, Friedman M. MR evaluation of rotator cuff pathology using T2weighted fast spin-echo technique with and without fat suppression. AJR Am J Roentgenol. 1996; 166:1061-65.

21.Szymanski C1, Staquet V, Deladerrière JY, Vervoort T, Audebert S, Maynou C. Reproducibility and reliability of subscapularis tendon assessment using CT-arthrography. Orthop Traumatol Surg Res. 2013;99(1):2-9.

\section{CONFLITO DE INTERESSES}

Os autores declaram não haver conflitos de interesse.

\section{AUTOR PARA CORRESPONDENCIA}

Aleixo Abreu Tanure

aleixotanure@gmail.com

Submetido em 07/12/2018

Aceito em 22/12/2018 